Please do not remove this page

RMIT

UNIVERSITY

\title{
Patterns of methamphetamine production among an international sample of methamphetamine 'cooks'
}

Puljevic, Cheneal; Zahnow, Renee; Benfer, Isabella; Winstock, Adam; Maier, Larissa; Barratt, Monica; Ferris, Jason

https://researchrepository.rmit.edu.au/esploro/outputs/9922036736301341/filesAndLinks?institution=61RMIT_INST\&index=null

Puljevic, C., Zahnow, R., Benfer, I., Winstock, A., Maier, L., Barratt, M., \& Ferris, J. (2020). Patterns of methamphetamine production among an international sample of methamphetamine "cooks." Journal of Drug Issues, 40(7), 1287-1293. https://doi.org/10.1111/dar.13205

Document Version: Accepted Manuscript

Published Version: https://doi.org/10.1111/dar.13205

Repository homepage: https://researchrepository.rmit.edu.au

(C) 2020 Australasian Professional Society on Alcohol and other Drugs

Downloaded On 2023/04/26 22:15:27 +1000 
4 Cheneal Puljević, $\mathrm{PhD}^{1}$

5 Renee Zahnow, $\mathrm{PhD}^{2}$

6 Isabella Benfer ${ }^{1}$

7 Adam R. Winstock, $\mathrm{PhD}^{3,4}$

8 Larissa J. Maier, $\mathrm{PhD}^{5}$

9 Monica J. Barratt, $\mathrm{PhD}^{6,7}$

10 Jason Ferris, $\mathrm{PhD}^{1}$

11

$12{ }^{1}$ Centre for Health Services Research, The University of Queensland, Brisbane, Australia

$13{ }^{2}$ School of Social Science, The University of Queensland, Brisbane, Australia

$14 \quad{ }^{3}$ University College London, Gower St, Bloomsbury, London, UK

$15 \quad{ }^{4}$ Global Drug Survey Ltd, London, UK

$16{ }^{5}$ Department of Clinical Pharmacology, University of California San Francisco, USA

$17{ }^{6}$ Social and Global Studies Centre, RMIT University, Melbourne, Vic, Australia

$18 \quad{ }^{7}$ National Drug and Alcohol Research Centre, UNSW Sydney, NSW, Australia

19

20 Corresponding author: A/Prof Jason Ferris, Centre for Health Services Research, Faculty of 21 Medicine, The University of Queensland, j.ferris@uq.edu.au, +61 731765530 
23 Introduction: The illicit manufacture of methamphetamine in clandestine laboratories is associated with significant risks to the community and environment. Currently little is known about clandestine laboratories or the individual "cooks" who operate them; current research

26 directly engaging with cooks is limited to three qualitative studies with small samples $(\mathrm{n}<24)$

27 of cooks based in the United States. This descriptive brief report starts to address this knowledge gap by exploring characteristics of an international sample of self-identified methamphetamine cooks.

Methods: Using data from the 2017 and 2018 Global Drug Surveys, we identified 125 individuals from 24 countries who reported past manufacture of methamphetamine. We explored respondents' socio-demographic characteristics and patterns of methamphetamine production using descriptive statistics.

34 Results: The majority of methamphetamine cooks were male (82\%) and Caucasian (70\%), and, contrary to previous studies, $43 \%$ were employed and $51 \%$ had at least a high school certificate. Cooks most commonly sourced precursors from pharmacies $(50 \%)$, followed by 37 friends (24\%). Almost half of the cooks (47\%) produced methamphetamine exclusively for self-consumption.

39 Discussion: The heterogeneous nature of the sample, and varying precursor sources, reflect

40 the limitations of existing regulations aimed at limiting methamphetamine production. These

41 findings point to the need for innovative and multi-faceted efforts aimed at reducing and

42 preventing the harms associated with methamphetamine manufacture. 
Methamphetamine is a highly addictive substance [1] that can be manufactured with relative ease in small domestic laboratories [2]. Recent global trends in drug seizures suggest a dynamic and growing market for methamphetamine in a number of countries [3]. Analyses of wastewater conducted in 37 countries between 2011 and 2017 show the highest levels of methamphetamine use in the USA, followed by Australia and New Zealand [4]. Methamphetamine is typically produced in clandestine laboratories (commonly referred to as clan labs) of varying sizes, ranging from "super" clan labs, which are large, organised labs capable of producing an average of 10 pounds $(4.5 \mathrm{kgs})$ per cycle, to smaller labs based in domestic residences that supply individual consumption [2]. Domestic clan labs were recently made infamous by the television series Breaking Bad. However, the television series failed to highlight the significant health risks clandestine laboratories pose to individuals and communities. As these clan labs involve the use of volatile and hazardous chemicals, often processed through makeshift equipment [5], fires and explosions can occur, causing significant injuries [6]. Further, the cooking process can result in the release of harmful reagents and by-products into the atmosphere, water and soil [7], posing significant public health risk to the environment and to the people who come into contact with clan labs.

The majority of people receiving injuries from clan labs are the individuals manufacturing the drugs [8], with common injuries including acute inhalation injuries, burns, or death following explosions $[8,9]$. These manufacturers often follow "recipes" found online, earning them the nickname of "cooks" $[10,11]$. As most clan labs are located in residential neighbourhoods [8], members of the public, including children, are also occasionally victim to chemical exposure associated with nearby clan labs [7, 9]. Clan labs are a public safety issue separate from individual drug use in the risks posed to the general non-using population [9], pointing to the need for effective strategies aiming to prevent this kind of methamphetamine production.

Due to difficulties engaging this population group in research, very little is known about cooks or their production methods; for example, we are aware of only three studies, all conducted in the USA-Missouri [12], Arkansas [13], Kentucky [13], and Oklahoma [14]which have engaged directly with small samples $(n<24)$ of current $[13]$ or former $[12,14]$ producers of methamphetamine. Other research examining methamphetamine manufacturers 
administrative records [15-17]. All studies describe cooks as characteristically young, male, Caucasian and unemployed, with low levels of education [12-17].

Information about cooks' production methods can inform prevention, intervention and harm reduction strategies, particularly those targeting the availability of precursors or tools used for manufacturing. Further, information about their demographic profiles may help to identify individuals at high risk of future methamphetamine manufacture, thus enabling prevention through early intervention. The current study starts to address some of these research needs. Drawing on survey data from a sample of 125 people who identified as methamphetamine cooks, this descriptive brief report explores patterns of methamphetamine production among this unique population.

\section{METHODS}

\section{Data source}

Data were drawn from the Global Drug Survey (GDS) [18], an annual, anonymous, online survey conducted in partnership with global media partners that captures respondents' lifetime and recent use of more than 150 drugs. The survey is open to anyone aged 16 and over who consents to participate, and is translated into multiple languages. Full details of the design, history and representativeness of the GDS are described elsewhere [18]. The study received ethical approval from University College London (11671/001), King's College London (PNM/14/15-17), The University of Queensland (2017001452/11671/001), and The University of New South Wales (HC17769).

Combined data from the 2017 and 2018 GDS were used for this study. A total of 119,108 and 130,761 people responded to the 2017 and 2018 GDS respectively. The sample described in this study was restricted to those who responded "yes" to the question "Have you ever made your own methamphetamine drugs?” For individuals who reported participating in both the 2017 and 2018 GDS surveys, we retained only their responses from the 2017 survey to avoid duplication.

\section{Analyses}

First we calculated descriptive statistics for socio-demographic variables, including sex, age, country of residence, geographic location (city/urban, regional or remote), educational achievement, employment status, current occupation, and drug use within the past year. Next we analysed their patterns of methamphetamine production, including common production methods, frequency of production, source of precursors, and the purpose 
107 of the manufactured methamphetamine (self-consumption / sale to others). Finally, we 108 compared cooks who manufactured methamphetamine for self-consumption, for sale to 109 others, or both.

\section{RESULTS}

111 The final sample of methamphetamine cooks included 125 respondents (66 from GDS 1122017 and 59 from GDS 2018) from 24 countries. Table 1 shows the socio-demographic 113 profile of the sample. The majority were male and Caucasian, and the cooks reported a mean 114 age of 30 (range 16-60). The largest number of the sample's respondents resided in the USA 115 (38\%), followed by Australia (10\%), New Zealand (9\%), Germany (7\%), and Canada (6\%).

116 One-third of respondents had a high school certificate, and almost half were currently

117 employed, with the most frequently reported occupation being a labourer or related 118 profession. Respondents reported a high level of substance use in the past 30 days, in

119 particular tobacco, methamphetamine, cannabis mixed with tobacco, and amphetamine.

Table 2 shows respondents' patterns of methamphetamine production for the full sample and for respondents from the five countries with the highest numbers of cooks. Approximately one-third of cooks had produced methamphetamine at least once within the past year. The most commonly-reported production method was using ephedrine/ pseudoephedrine as a precursor, followed by a reduction of hydroiodic acid and red phosphorous, although these processes often form two parts of the same method. Pharmacies were the most common source of precursors, followed by friends. Almost half of respondents described manufacturing methamphetamine for self-consumption only.

Supplementary Table 3 shows the socio-demographic characteristics of respondents who reported manufacturing methamphetamine for self-consumption, for sale to others, or

130 both. Although small sample sizes in each group and missing data limit our ability to draw accurate comparisons between the groups, those who reported manufacturing exclusively for self-consumption appear to be similar in age, ethnicity, level of education and employment status compared to the overall sample and those who manufacture for both self-consumption and sale to others.

Our analyses of an international sample of self-identified methamphetamine cooks

137 provides new information about individuals involved in methamphetamine manufacture. Our

138 findings show that methamphetamine cooks are heterogeneous in both their socio- 
demographic characteristics and their adopted method of methamphetamine synthesis. Here we report two key findings that add to the current literature.

First, in contrast to previous single-region studies describe methamphetamine cooks as characteristically unemployed, and with limited education [12-17], our sample contains individuals representing a wider range of socio-demographic characteristics. The majority of individuals in our sample had completed high school and were employed and many were in professional or managerial roles. This finding implies that clandestine laboratories may exist in many types of neighbourhoods, including higher socioeconomic areas.

Our second key finding is that respondents most commonly described manufacturing methamphetamine for self-consumption only, suggesting small-scale manufacture within domestic residences, while only a small percentage of respondents reported manufacturing methamphetamine exclusively for sale to others. This finding reflects those from literature describing cannabis cultivators primarily growing cannabis for personal use [19, 20], although the cultivation of cannabis is arguably less risky in terms of risk of exposure to hazardous chemicals or explosions. Domestic production of methamphetamine is of

154 particular concern when considering the public health harms associated with clan labs located 155 in residential areas, such as fires and explosions [7, 11], or exposure of members of the public to hazardous chemicals $[7,9,11]$. Further, the sporadic and diverse nature of this type of manufacture makes it particularly difficult to locate and prevent [2]. In addition to the provision of traditional drug education and treatment services aimed at reducing demand for methamphetamine $[1,2]$, there may be benefit in training third parties who have routine access to residences (such as realtors or cleaners) to detect and report suspicious activity related to methamphetamine manufacture.

A number of legislative controls have been implemented internationally in an attempt to prevent methamphetamine manufacture (see Stoneberg et al for a review [2]), such as

164 limits on the sales and supply of precursors required for methamphetamine production [21, 22]. While these regulations have previously demonstrated effectiveness in reducing indicators of methamphetamine supply [21-23], many cooks are now able to synthesise precursors from processed products [10], and international efforts to disrupt illicit drug supply, trafficking and manufacture have met with limited success [2]. These regulations also may be less effective in preventing methamphetamine manufacture by cooks producing methamphetamine exclusively for individual consumption. As a consequence, our results 
171 provide support for innovative and multi-pronged approaches to prevent unregulated

172 methamphetamine manufacture and related harms, such as exposure to harmful chemicals, or

173 injuries from fires or explosions. There also may be merit in considering targeted outreach

174 approaches (for example, campaigns promoting less risky methods of cooking), or even the

175 safe supply of methamphetamine within a legal, regulated context — an approach with the

176 potential to reduce harms among a group who often experience various forms of disadvantage

177 and marginalisation [24, 25]. Given many cooks indicate manufacturing only for self-

178 consumption, these strategies may also reduce potential community harms associated with

179 small-scale methamphetamine manufacture. Further research is also required on this topic, in

180 particular regarding motivations for methamphetamine production, whether there is any

181 significant difference between purchased and produced product in terms of perceived quality

182 or value for money, and effective interventions or strategies to prevent unregulated

183 methamphetamine manufacture and related harms.

184 Our study benefits from its unique international sample and the GDS survey's 185 anonymity, and highlights that people who manufacture methamphetamine, for personal use

186 or supply, are prepared to complete online surveys about their manufacturing practices.

187 However, our findings are subject to some limitations. First, our data are not necessarily

188 representative of individuals who manufacture methamphetamine; the GDS is a self-select,

189 self-report survey, and only included individuals who had used a drug within the past 12

190 months. As a result of this and the sample size, analyses were restricted to descriptive

191 statistics. In addition, ethical considerations necessitated designing the questions as optional

192 to complete, resulting in missing data. Third, participants were limited to three response

193 options when describing the purpose of methamphetamine production (self-consumption, sale

194 for others, or both), excluding those who may have manufactured methamphetamine for

195 social supply or other purposes. Finally, we are unable to provide details about the context

196 within which individuals manufactured methamphetamine (e.g. the clan lab's size or

197 location), or respondents' motivations behind production.

\section{Conclusions}

199 In this study we highlighted two key findings: many individuals who manufacture

200 methamphetamine are employed and have finished high school, and almost half of cooks

201 reported manufacture for self-consumption only. While our study provides some unique

202 insights into the characteristics and behaviours of methamphetamine cooks, more research is 
203 required to inform policies aimed at both prevention of and intervention in methamphetamine 204 manufacture, and the potential impact of the safe supply of methamphetamine. 
206 1. Degenhardt L, Sara G, McKetin R, Roxburgh A, Dobbins T, Farrell M, et al.

207 Crystalline methamphetamine use and methamphetamine-related harms in Australia. Drug

208 Alcohol Rev. 2017;36(2):160-70.

209 2. Stoneberg DM, Shukla RK, Magness MB. Global Methamphetamine Trends.

210 International Criminal Justice Review. 2018;28(2):136-61.

$2113 . \quad$ United Nations Office on Drugs \& Crime. World Drug Report 2018. Vienna,

212 Australia: United Nations Office on Drugs and Crime; 2018.

213 4. González-Mariño I, Baz-Lomba JA, Alygizakis NA, Andrés-Costa MJ, Bade R,

214 Barron LP, et al. Spatio-temporal assessment of illicit drug use at large scale: evidence from

2157 years of international wastewater monitoring. Addiction. 2020;115(1):109-20.

216 5. Abdullah AFL, Miskelly GM. Recoveries of trace pseudoephedrine and

217 methamphetamine residues from impermeable household surfaces: Implications for sampling

218 methods used during remediation of clandestine methamphetamine laboratories. Talanta.

219 2010;81(1-2):455-61.

2206 6oper JD. Drug-endangered children and the manufacture of methamphetamine.

221 School Nurse News. 2007;24(2):27-9.

222 7. Caldicott DG, Pigou PE, Beattie R, Edwards JW. Clandestine drug laboratories in

223 Australia and the potential for harm. Australian and New Zealand Journal of Public Health.

224 2005;29(2):155-62.

225 8. Australian Government. enHealth Guidance on: Clandestine Drug Laboratories and

226 Public Health Risks. 2017.

227 9. Wright J, Edwards J, Walker S. Exposures associated with clandestine

228 methamphetamine drug laboratories in Australia. Reviews on Environmental Health.

229 2016;31(3):329-52.

230 10. Vidal S, Décary-Hétu D. Shake and Bake: Exploring Drug Producers' Adaptability to

231 Legal Restrictions Through Online Methamphetamine Recipes. Journal of Drug Issues.

232 2018:0022042617751685.

233 11. Burgess JL, Chandler D. Clandestine drug laboratories. In: Greenberg MI, Hamilton

234 RJ, Phillips SD, McCluskey GJ, editors. Occupational, Industrial, and Environmental

235 Toxicology (2nd Ed). St Louis, Missouri: Mosby; 2003.

236 12. Jenkot R. "Cooks are like Gods": Hierarchies in methamphetamine-producing groups.

237 Deviant Behav. 2008;29(8):667-89.

238 13. Sexton RL, Carlson RG, Leukefeld CG, Booth BM. Patterns of illicit

239 methamphetamine production ("cooking") and associated risks in the Rural South: An

240 ethnographic exploration. Journal of Drug Issues. 2006;36(4):853-76.

14. Shukla RK. Methamphetamine: A Love Story. Oakland, CA: University of California Press; 2016.

243 15. Danks RR, Wibbenmeyer LA, Faucher LD, Sihler KC, Kealey GP, Chang P, et al.

244 Methamphetamine-Associated Burn Injuries: A Retrospective Analysis. Journal of Burn Care 245 \& Rehabilitation. 2004;25(5):425-9.

246 16. Thrasher DL, Von Derau K, Burgess J. Health effects from reported exposure to

247 methamphetamine labs: a poison center-based study. Journal of Medical Toxicology.

$248 \quad 2009 ; 5(4): 200-4$.

249 17. Warner P, Connolly JP, Gibran NS, Heimbach DM, Engrav LH. The

250 methamphetamine burn patient. J Burn Care Rehabil. 2003;24(5):275-8. 
19. Potter GR, Barratt MJ, Malm A, Bouchard M, Blok T, Christensen AS, et al. Global patterns of domestic cannabis cultivation: sample characteristics and patterns of growing across eleven countries. The International journal on drug policy. 2015;26(3):226-37. 20. Decorte T. Small Scale Domestic Cannabis Cultivation: An Anonymous Web Survey among 659 Cannabis Cultivators in Belgium. Contemporary Drug Problems. 2010;37(2):34125970.

260 21. McKetin R, Sutherland R, Bright DA, Norberg MM. A systematic review of methamphetamine precursor regulations. Addiction. 2011:1-14.

262 22. Ferris J, Devaney M, Mazerolle L, Sparkes-Carroll M. Assessing the utility of Project 263 STOP in reducing pseudoephedrine diversion to clandestine laboratories. Trends and Issues 264 in Crime and Criminal Justice. 2016;509(March):1-7.

265 23. Mazerolle L, McGuffog I, Ferris J, Chamlin MB. Pharmaceutical sales of

266 pseudoephedrine: the impact of electronic tracking systems on methamphetamine crime 267 incidents. Addiction. 2017;112(3):468-74.

268 24. Fleming T, Barker A, Ivsins A, Vakharia S, McNeil R. Stimulant safe supply: a 269 potential opportunity to respond to the overdose epidemic. Harm Reduction Journal. $270 \quad 2020 ; 17(1): 6$.

271 25. Greer A, Ritter A. The legal regulation of drugs and role of government: Perspectives 272 from people who use drugs. Drug Alcohol Depend. 2019;206:107737. 